\title{
Redescubriendo el paradigma Ubuntu en educación: Contribuciones epistemológicas en Educación Comparada e Internacional
}

\author{
Lázaro Herrero, Luján \\ Facultad de Educación, Universidad de Salamanca, España \\ lujan@usal.es
}

\section{Resumen}

Este trabajo plantea la necesidad de incluir paradigmas alternativos en los escenarios tanto, teóricos como metodológicos, propios de la Educación Comparada e Internacional, que sean menos positivistas, eurocéntricos, clasistas e individualistas.

En la búsqueda de comparación educativa, la hegemonía de análisis lineales, unidireccionales, credencialistas y centrados en sistemas de clasificación global, han caracterizado el devenir científico en el campo que nos ocupa, aspecto que nos lleva a cuestionarnos ¿qué estamos comparando?, ¿qué teorías estamos utilizando para entender los procesos educativos? y ¿qué políticas y prácticas educativas se están transfiriendo? Así pues, a través de estas líneas, defendemos la idea de una epistemología comparada necesitaría de enfoques holísticos, humanistas, centrados en el ser humano, que reconozcan la conexión e interdependencia entre las personas, y que discutan las relaciones asimétricas entre Occidente y el Sur global, así como los resultados de las mismas.

Es por ello que, el objeto de esta comunicación es el análisis del paradigma filosófico Ubuntu en cuanto a su significado de humanidad compartida, así como, el lugar que toma en la educación. Ubuntu es cosmovisión y por ello, se presenta como un marco de referencia colectiva, útil en la definición de relaciones en y entre fronteras locales y globales, de aplicación no sólo en territorio africano sino a nivel mundial. Presentamos los postulados educativos de este modelo paradigmático e intentamos establecer una vía convergente de investigación que puede contribuir al enriquecimiento y renovación epistemológica de la educación comparada e internacional. Todo ello, en un momento en el que ésta, la educación comparada, sigue siendo objeto de intensos debates, en los que se cuestiona su sentido y orientación como ciencia, al mismo tiempo que se destacan los desafíos a los que se enfrenta.

\section{Abstract}

This work raises the need to include alternative paradigms in the scenarios, both theoretical and methodological, typical of Comparative and International Education, which are less positivist, Eurocentric, classist and individualist.

In the search for educational comparison, the hegemony of linear, unidirectional, credentialist analyses centred on global classification systems have characterized the scientific evolution of the field in question, an aspect that leads us to ask ourselves: What are we comparing? What theories are we using to understand educational processes? What educational policies and practices are being transferred? Thus here we defend the idea of a comparative epistemology that would entail holistic, humanistic, person-centred approaches to recognize the connections and interdependence among people, and to discuss the asymmetric relations between the West and the global South, as well as the results thereof.

The aim of this communication is therefore the analysis of the Ubuntu philosophical paradigm regarding its meaning of shared humanity, as well as the place it occupies in education. Ubuntu is a worldview and therefore it presents itself as a collective frame of reference, useful in the definition of relationships in and between local and global borders, applicable not only in Africa but worldwide. We present the educational postulates of this paradigmatic model and try to establish a convergent research path which can contribute to the enrichment and epistemological renewal of comparative and international education. All this, at a time when comparative education continues to be the subject of intense debates, in which its meaning and orientation as a science is questioned, while at the same time highlighting the challenges it faces.

Palabras clave: Paradigma Ubuntu, epistemología, educación comparada, países colonizados, educación humanística.

Keywords: Ubuntu Paradigm, epistemology, comparative education, colonial countries, humanistic education. 
"umuntu ngumuntu ngabantu" Una persona es sólo persona a través de su relación con los demás.

El significado de «Ethos» colectivo del término Ubuntu ha sido a lo largo de la historia popularizado por varios autores. Fue quizás el periodista y novelista Jordan Kush Ngubane el que, en la década de 1950, situó el concepto en la vanguardia filosófica. Más recientemente, figuras como Nelson Mandela y el teólogo Desmond Tutu- ambos, Premios Nobel de la Paz- han apelado a Ubuntu para la articulación de la inclusión y la igualdad, es más, es posible que la mayor experiencia de aplicación de Ubuntu fuera la Comisión de Verdad y Reconciliación presidida por Tutu, establecida en Sudáfrica después de la abolición del apartheid.

El lugar de Ubuntu en la educación fue ampliamente analizado en un número especial de la International Review of Education, en el año 2016, que surgió a partir de las aportaciones de la $59^{\mathrm{a}}$ conferencia anual de la Sociedad Comparada e Internacional (CIES) celebrada en 2015 en Washington D.C, y cuyo tema fue: «iUbuntu! Imaginar una educación humanista en todo el mundo». Explica Oviawe (2016), encargado de coordinar el monográfico, que el objeto de este foro académico fue el de proporcionar una visión crítica de los sistemas educativos europeos que se establecieron en sociedades colonizadas del Sur global, incluida África. Un encuentro que sirvió para vislumbrar y denunciar el hecho que la educación fuera conceptualizada, dibujada y aplicada como un instrumento de dominio. Ali A. Abdi fue uno de los académicos que participó en dicho evento, y relata en un reciente trabajo (2018) cómo ese foro de conocimiento le sirvió para darse cuenta de que podría estar surgiendo una perspectiva catalizadora de aspectos africanos incorporados a la investigación, lo que situaba a la filosofía africana Ubuntu en una posición emergente.

Redescubrir el paradigma Ubuntu significa otorgar a esta filosofía una posición de primera línea, en el sentido de volver a conocer sus postulados, quizás relegados a un segundo plano, y situarlos en el epicentro de la investigación. Se presenta pues, un marco teórico y cultural que enfatiza las conexiones humanas y la interdependencia a la vez que, va a guiar la investigación y la práctica del campo de la Educación Comparada e Internacional, ECl.

En este sentido, el trabajo presentado por Assie'-Lumumba (2016b) publicado en la Comparative Education Review, relaciona directamente el paradigma Ubuntu con la $\mathrm{ECl}$ y abre una puerta hacia el conocimiento de las nuevas teorías relacionales transformativas así como, reivindica la utilización de metodologías que comprendan la educación en las sociedades anteriormente colonizadas. Reclama el espacio educativo, en este caso, para África, que le permita evolucionar cultural e intelectualmente y frenar los efectos del legado colonial, definidos por la fuerza del eurocentrismo y la injusticia global propagada.

Es por ello, que esta comunicación, coge el guante lanzado por la autora citada anteriormente y pretende seguir en la misma línea de conocimiento que profundice en el significado de Ubuntu y muestre su relación con la ECI. Por ello, a través de estas líneas, defendemos la idea de una epistemología comparada necesitaría de enfoques holísticos, humanistas, centrados en el ser humano, que reconozcan la conexión e interdependencia entre las personas, y que discutan las relaciones asimétricas entre Occidente y el Sur global, así como los resultados de las mismas.

El trabajo se articula a través de tres epígrafes. En el primero se indaga en el significado de Ubuntu en cuanto a espíritu, ética y valor así como en el papel que toma en un momento crucial en devenir político de la República de Sudáfrica. El segundo apartado lo dedicamos a mostrar los desafíos a los que, a nuestro entender, se enfrenta la $\mathrm{ECl}$ en un momento en el que se cuestiona su sentido y orientación como ciencia a la vez que se le pide una adaptación ontológica a los cambios. Por último, situamos el lugar que Ubuntu ocupa en la educación, en contraposición a las tesis educativas del sistema globalizador neoliberal, imperante durante tiempo en las sociedades colonizadas, denunciar su fracaso como proyecto educativo de humanización y vislumbrar la posibilidad real de una educación humanística global apoyada en la educación humanística africana de Ubuntu. 


\section{1. ¿QUÉ ES UBUNTU? ETHOS COLECTIVO}

\section{“(...) there is a need for understanding but not for vengeance, a need for reparation but not for retaliation, a need for Ubuntu but not for victimization" «(...) hay una necesidad de comprensión, pero no de venganza, una necesidad de reparación, pero no de represalias, una necesidad de ubuntu, pero no de victimización» (Constitución de la República de Sudáfrica, 1993, Acta 200, epílogo)}

La transición democrática en Sudáfrica estuvo cargada de importantes connotaciones emotivas (González Marrero, 2012) que, de alguna manera, le definen como un modelo de democratización singular, del que se ha hecho eco reiteradamente la ciencia política contemporánea. El paso de la Sudáfrica del apartheid- régimen político autoritario, colonialista y radical- propagador de segregación racial y de una "pigmentocracia dominada por los blancos» (Lyon, 1994, p.271) a un sistema de convivencia armónica implicó, no sólo el nacimiento de una constitución y la convocatoria de unas elecciones libres, sino la necesidad de reconstrucción de un país fragmentado política y socialmente.

La reforma constitucional de 1993, a partir de la cual todos los ciudadanos sudafricanos, independientemente de su raza, son iguales ante la ley, marcó un hito en este proceso de conversión. Precisamente, hemos abierto este epígrafe recogiendo un pequeño fragmento del texto constitucional en el que aparece la referencia a Ubuntu que si bien, no se explica en qué consiste, sí se aprecia cómo el espíritu del mismo, invade toda la orden reglamentaria. El extracto citado, refleja la necesidad imperante, a partir de la cual, la sociedad sudafricana tiene de mirar hacia delante, de tender puentes hacia la construcción a la vez que debe reparar heridas y unir a la ciudadanía. En definitiva, apela a la necesidad de Ubuntu y nos hace entender que éste va a ser una herramienta fundamental de reconciliación y construcción, un elemento clave en la superación de las divisiones del pasado.

No es la primera vez que el término Ubuntu aparece en la escena sociopolítica del continente africano. Gade (2011) sitúa las primeras referencias literarias al mismo a partir del año 1846 ligadas al estudio de culturas indígenas africanas, a la vez que, indica que el vocablo puede tener diferente significado en función del territorio y cultura contemplada. Añade que, la consideración de ubuntu como filosofía y humanismo africano emerge en la segunda mitad de los 90'y, demuestra en su análisis, cómo «ubuntu» llega a ser un objeto de especial interés y consideración durante los periodos políticos de transición (...) en Zimbabue y Sudáfrica» (p. 303).

La palabra Ubuntu tiene su origen en los dialectos locales del sur de África. Proviene de la lengua bantú (unidad lingüística de diferentes lenguas africanas) y relacionada con la expresión zulú: "umuntu ngumuntu ngabantu" y en mashi sería: "O'muntu ajirwa n'owabo". Todas ellas, vienen a expresar la idea de que una persona se hace humana a través de otras personas.

Llegados a este punto cabría cuestionarse ¿qué es ubuntu? Y para ello, nos vamos a apoyar en la investigación realizada por el profesor Chistian Gade (2012), el cual, hace una distinción analítica entre dos grupos de respuestas obtenidas a partir de la pregunta (¿qué es para vosotros ubuntu?) que planteó a académicos sudafricanos de ascendencia africana. Aunque hay variaciones internas en las respuestas de un mismo grupo, señala que existe una constante común en el primer grupo a partir de la cual, Ubuntu es definido como una cualidad moral de la persona mientras que, en el segundo, se precisa como un fenómeno (una filosofía, una ética, una cosmovisión y un humanismo africano) según el cual, las personas están interconectadas. Destaca igualmente el autor cómo el concepto de «persona» ocupa una posición de vital importancia respecto a lo que los encuestados entienden por ubuntu en ambos grupos. Así, el primer grupo, hace alusión a una cualidad moral de la persona y sólo si eres persona puedes poseer esa condición ética, que es individual. Para el segundo grupo, es a través de la filosofía ubuntu, donde las personas se conectan entre sí y obviamente, si no eres persona, no vas a tener posibilidad ni vas a ser parte de esa conexión.

El intento de conceptualización del término que nos ocupa, nos lleva a constatar cómo ubuntu se contempla como una filosofía comunitaria, un código ético, que «(...) implica el reconocimiento incondicional de la singularidad y la pluralidad de cualquier ser viviente en el cosmos «(Botero Gómez p.87), lo que supone que (...) el individuo no es independiente del colectivo; más bien, la relación entre una persona y su comunidad es recíproca, interdependiente y mutuamente beneficiosa» (Oviawe, $p$. 3). Añade Sayers (2012) que «ubuntu es una antigua forma de vida que personifica la generosidad, calidez, inclusión y solidaridad que aún son típicas en muchas comunidades africanas». (p. 7). Ubuntu «significa humanidad o humano» (Cillerss, 2017, p.67) y para la ética africana, la única forma de desarrollar la propia humanidad es relacionarse con los demás de una manera positiva. 
De estas concepciones, se desprende una visión de comunidad propia de la moralidad africana y que, dista de la acuñada en occidente (Metz \& Gaie, 2010). Hacer, buscar comunidad y vivir en armonía sería la forma de relacionarse. No se trataría de adoptar una posición de sumisión al grupo, de adhesión a sus normas dominantes, sino de encontrar un camino interactivo de respeto y desarrollo de la comunidad en concordancia, a través de unas relaciones sociales cercanas y comprensivas dentro del grupo. Según Kakozi (2011) la aplicación práctica de estos pensamientos se puede apreciar, por ejemplo, en la proyección que sobre la familia se tiene en África ya que «ésta no se reduce solamente a papá, mamá e hijos, sino que se extiende a los demás miembros lejanos de la familia. En efecto, es muy común en África escuchar a alguien presentar a su prima o primo, como su hermana o hermano» (p.48). El concepto que se tiene de comunidad y de bien común hace que la persona se sienta arropada:

Una persona en Ubuntu está abierta y disponible a los demás, no se siente amenazada o está en competencia, como en el sistema capitalista. Parte de una adecuada seguridad en sí misma que proviene de saberse perteneciente a un todo mayor, común, donde se incluyen a sus ancestros, la naturaleza y animales, además de todos los seres humanos desde su familia, tribu y otras comunidades, tanto de los sujetos vivos, como sus ancestros, así como los sujetos naturales (Cárdenas, 206, p. 563).

Por encima de todo, Ubuntu representa la reconciliación, está cargado de un potencial ávido para tender puentes, crear unidad entre las personas, las culturas, los espacios y los tiempos.

\title{
2. DESAFÍOS DE LA EDUCACIÓN COMPARADA E INTERNACIONAL EN UN MARCO DESCOLONIZADO
}

\begin{abstract}
Readers might wonder how $\mathrm{CIE}$, which celebrates and tries to understand the diversity of education around the world, can continue to be influenced by colonial histories and Eurocentrism.

Los lectores podrían preguntarse cómo la $\mathrm{ECl}$, que aclama e intenta comprender la diversidad de la educación en todo el mundo, puede seguir siendo influenciada por las historias coloniales y el eurocentrismo. (Takayama, Sriprakash y Connell, 2016, p.1).
\end{abstract}

Takayama, Sriprakash y Connell (2016) en su artículo titulado «hacia una Educación Comparada e Internacional Poscolonial», hacen una llamada a la comunidad comparativista y subrayan la necesidad de efectuar una profunda reflexión colectiva respecto a la forma en que el conocimiento de $\mathrm{ECI}$ se está produciendo y cómo transita a nivel global. Pretenden los autores mostrar y criticar la perdurable colonialidad existente en el campo, así como hacer una invitación hacia la implementación de una práctica científica que escape a los dominios epistemológicos del Norte global:

Cuando figuras tan influyentes como Marx, Durkheim y Weber, y estudiosos más contemporáneos como Luhmann, Foucault, Bourdieu, Giddens y Beck, muestran una falta de comprensión de la colonialidad y, por lo tanto, una comprensión defectuosa y parroquial ${ }^{1}$ de la modernidad, y los estudiosos en $\mathrm{ECl}$ que han utilizado sus teorías, también se ponen en tela de juicio. Cuando se considera que los modelos científico-sociales de la globalización son eurocéntricos, y cuando se reconoce que las comprensiones defectuosas y parroquiales de la modernidad se han proyectado sobre el resto del mundo, un momento de profunda reflexión parece estar justificado para nuestro campo (Takayama, Sriprakash y Connell ,2016, p.4)

Encuadra García Ruíz (2012b) las teorías poscolonialistas en un marco de pensamiento posestructuralista, a partir del cual, el papel y significado de los elementos culturales y locales en el proceso de cambio educativo, toman especial relevancia. Añade la autora que «la teoría postcolonial (...) revela una gran sensibilidad por las cuestiones del contexto, la cultura y la diferencia. (...). La teoría post-colonial posee un gran potencial para clarificar las relaciones entre la globalización, el contexto y la diferencia» (p.135). Destacan Espejo, Beltrán, Lázaro y García (2014) igualmente, la relevancia que toman los enfoques poscoloniales, a la vez que, los sitúan en dos niveles diferentes de interpretación.

\footnotetext{
${ }^{1}$ En este caso, el término parroquial hace alusión al hecho de que los trabajadores, investigadores se identifican no tanto con su profesión sino con las organizaciones para las que trabajan.
} 
Por un lado, aquella posición que define esta orientación desde el punto de vista de las identidades culturales y su relación con la escuela y, por otro, el que los enmarca en un contexto de relativismo cultural, a partir del cual se ponen en duda la primacía de los valores formados en el «Oeste».

Como sabemos, el campo de la $\mathrm{ECl}$ estuvo dominado en el pasado por los imperativos de Europa y América del Norte. Rui (2010), nos recuerda la importancia que tiene en la realización de estudios comparados, el conocimiento de las tendencias y cómo estás van cambiando en búsqueda de perspectivas alternativas que se distancien de ese predominio científico que ha impregnado el campo:

Muchos estudiosos tuvieron su origen en Occidente, mientras que muchos investigadores no occidentales fueron entrenados en instituciones occidentales. Sus intereses de investigación fueron, en su mayoría, motivados por las preocupaciones normativas de mejorar sus propios sistemas educativos y los deseos modernistas de ayudar al «sur» a lograr el desarrollo (Rui, 2010, p.317).

Es por ello, que esta primacía epistemológica occidental viene siendo cuestionada por posiciones alternativas que, se sitúan próximos a la ciudadanía mundial del sur. Destaca Collado (2016) la existencia emergente de una "epistemología del sur» promovida "entre pensadores que reivindican la presencia del sur global para transgredir el paradigma de imposición epistémica occidental de la sociedad globalizada actual» (p. 141) y añade, parafraseando a Boaventura de Sousa Santos que esta epistemología «se asienta en tres orientaciones: aprender que existe el Sur; aprender a ir para el Sur; aprender a partir del Sur y con el Sur» (p.141).

El desarrollismo es uno de los postulados que identifica Garrido (2012) como básicos en el devenir de los estudios comparados tras la segunda guerra mundial y se refiere al mismo como el «mito del desarrollo». El estudio de sistemas nacionales de educación en busca de eficacia, ha llevado a los comparativistas a desempeñar un papel relevante en el establecimiento de sistemas educativos en sociedades colonizadas bajo el manto de un paradigma modernista de progreso y desarrollo. Abdi (2015) mantiene que la presencia de la retórica del desarrollo ha descontextualizado socioculturalmente a los individuos de estas sociedades, doblegados a un sistema individualista que les insta a ser competitivos entre ellos en la búsqueda de una prosperidad definida por parámetros modernos. Competencia y desigualdad sería el resultado de todo ello.

Explica de una manera muy clarificadora Bray (2010) el hecho de que, los encargados del diseño de políticas educativas buscan prácticas «dignas de tener en cuenta» y lo hacen en un plano cargado de prejuiciosos que van a condicionar el tipo de lugar elegido para llevar a cabo la investigación. Así pues, el idioma del país, los lazos políticos establecidos y las percepciones sobre jerarquía que se tengan, van a condicionar la búsqueda de comparación, así como el territorio y unidad objeto de la misma:

(...) países que ya han alcanzado el desarrollo económico tienden a observar a aquellos que han logrado un nivel similar de progreso. Funcionarios de países industrializados no indagan, en general, ideas y modelos en países emergentes, aunque en ocasiones deberían hacerlo. (Bray, 2010, p. 42).

Va más allá Benjamín Piper (2016) cuando sentencia categóricamente que el campo de la Educación Internacional está roto y, señala directamente a los actores principales del desarrollo educativo, como los causantes de esa fractura, acusándolos de «no cuestionar suficientemente las dinámicas de poder que sustentan el desarrollo de la educación» (p. 101). Resalta el «mal» de una hegemonía occidental a partir de la cual se generan desequilibrios de poder que, conducen a que las prácticas de desarrollo educativo se promuevan desde un plano unidireccional en el que los expertos no tienen en cuenta el contexto, no se preocupan por conocer el lugar y no aprecian la cultura local.

En un intento de arrojar luz en el ámbito de la educación internacional y el desarrollo educativo, continúa el autor anteriormente mencionado, sugiriendo prácticas que podrían contribuir al avance del campo, así como al freno de la expansión de programas educativos dominantes. Así, resalta la necesidad de cultivar la humildad entre los técnicos de la educación, que sean éstos capaces de apreciar lo positivo de la experiencia que puedan tener los técnicos nativos como grandes conocedores de la cultura local. Revisar y flexibilizar los procesos de investigación para dar cabida a profesionales femeninas, a expertos locales y minorías étnicas. Todo ello, requería un cambio de la actitud de los profesionales y, aseguraría una armonía relacional entre Occidente y el Sur Global. 
Se encuentra igualmente la $\mathrm{ECl}$ evocada a revisar y cuestionar las relaciones con su objeto de estudio ya que no tiene cabida que éste (su objeto) esté delimitado por el Estado nacional. Sabemos que la educación comparada «nació en una época en que los estados-nación eran los actores principales de las políticas y prácticas educativas» (Acosta y Ruiz, 2015, p.15). Sin embargo, «la globalización ha acentuado la importancia del lugar y la ubicación» (García Ruiz, 2012 a, p.58), «los llamados fenómenos globales se extienden más allá de regiones y naciones» (López, 2008, p. 5). Y así lo constata Larser (2010) al referiré al «giro espacial» en las ciencias sociales lo que hace desafiar al área epistemológica «a desarrollar nuevas vías de teorización respecto a espacios, lugares y ubicaciones en el campo comparativo» (p.6).

Así pues, destacan Takayama, Sriprakash y Connell (2016) cómo la ECI se encuentra en un momento de desafío de sus puntos de vista convencionales, a partir del cual, se están proponiendo proyectos que, descentralizan el Norte global en la búsqueda y proyección de conocimiento. Y así lo recogen los propios autores:

Los estudiosos contemporáneos han propuesto proyectos de conocimiento que descentran el Norte global en la producción de conocimiento, socavan las relaciones desiguales de poder que naturalizan la división intelectual del trabajo, provincializan la ontología y epistemología universal que sustentan el conocimiento oficial y revalorizan los conocimientos que han sido subyugados por la hegemonía global (p.13).

Estos cambios iniciados se pueden observar, por ejemplo, en el papel que tiene el estudio de las culturas, que retoman una posición céntrica como unidad de comparación, ya que, «pocos podrían negar que los factores culturales están asociados con algunos aspectos de la educación y la inluyen» (Mason, 2010 , p. 207). Conocer y comprender la cultura local es esencial para garantizar la implementación de programas educativos, por lo que desconocer u obviar aspectos culturales de la comunidad, va a desembocar en el fracaso de éstos. Reconocer la delicada herencia que emana en el núcleo de cada cultura nos lleva a entender que uno no puede comprender las dinámicas educativas si no toma en cuenta los entornos sociales y culturales.

\section{EI LUGAR DEL PARADIGMA UBUNTU EN LA EDUCACIÓN. POSIBILIDADES EPISTEMOLÓGICAS EN EL ÁMBITO COMPARADO E INTERNACIONAL}

«(...) there is a place for an Ubuntu philosophy in education both in Africa and around the world» «(...) hay un lugar para una filosofía Ubuntu en educación, tanto en África como alrededor del mundo» (Oviawe, 2016, p.2-3).

Figueras (2010) en su libro, en el que indaga sobre cómo fue posible que Sudáfrica celebrara un evento de tal magnitud como puede ser un Mundial de Fútbol-2010- siendo un país gobernado durante mucho tiempo por un régimen de minoría blanca opresor y generador de racismo-, muestra este hecho como un ejemplo de entendimiento y unión social. En su relato contempla que ubuntu, es una filosofía que se puede aplicar al deporte, a la empresa, a la vida cotidiana y... ¿a la educación? Esta es la cuestión que desde este trabajo nos planteamos. ¿Es posible una educación ubuntu en la actualidad?, ¿puede la filosofía ubuntu proporcionar perspectivas epistemológicas alternativas en el campo de la ECl?

La filosofía Ubuntu promueve el humanismo que, conceptualiza al mundo como un sistema complejo e interdependiente. Tal y como mostramos en la figura 1, Ubuntu fomenta una educación humanística que enfatiza las contribuciones colectivas para el aprendizaje de todos. El fin es el avance hacia la unidad. Sería una educación que conduciría al desarrollo social, que mira hacia nuevas posibilidades de bienestar social. Una filosofía y práctica de la dignidad humana. 
Desarrollo humano y progreso social requieren de un avance de las capacidades humanas que vendría de la mano de un conocimiento superior. Nos sitúa Assié-Lumumba (2016b) ante la noción de conocimiento superior en un contexto global, y lo define «como la suma de la sabiduría colectiva adquirida a lo largo de la historia y enriquecida con nueva información, que sirve como una brújula de la sociedad» (p.11). Requiere, que la persona esté abierta y disponible para los demás, sin que se sienta amenazada por las capacidades de los otros ya que, «la interdependencia humana y la coexistencia parecen ser la forma más apropiada de articular el concepto» (waghid, 2018, p. 56) de educación humanística Ubuntu.

Figura 1. Educación humanística de Ubuntu.

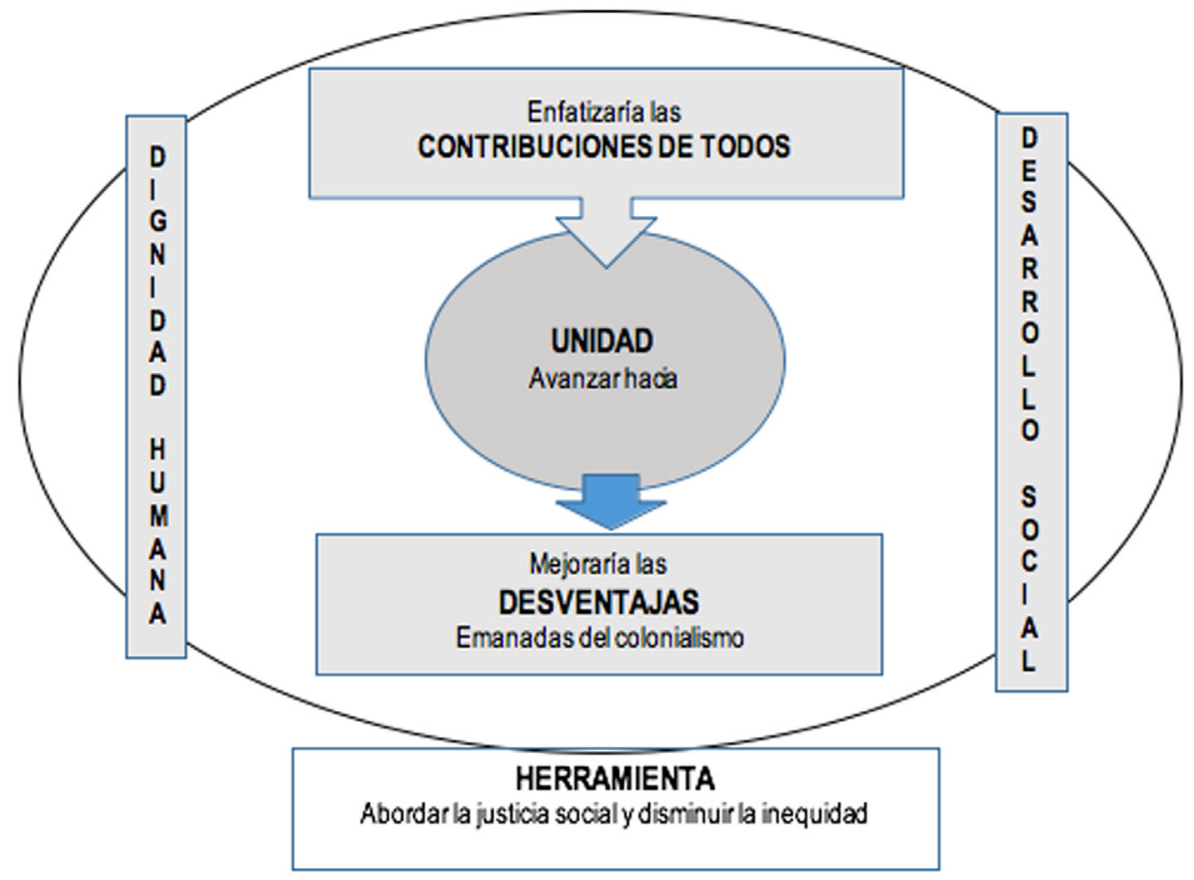

Fuente: elaboración propia a partir de Abdi (2015).

La búsqueda de una educación humanística en Ubuntu pretende frenar el predominio de tendencias dominantes procedentes de un enclave euronorteamericano a partir del cual la educación ha sido utilizada como instrumento para crear ciudadanos "colonizados». La figura 2 ilustra el contraste existente entre los postulados de la educación modernista propia de la globalización neoliberal y los que establece la filosofía Ubuntu que, acepta de manera incondicional la humanidad plena del otro y de todos. Encuentra la educación humanística de Ubuntu su naturaleza en el diálogo entre pueblos y civilizaciones, busca el consenso y la convergencia de ideas. 
Figura 2. Educación humanística Ubuntu frente a Educación globalizadora neoliberal.

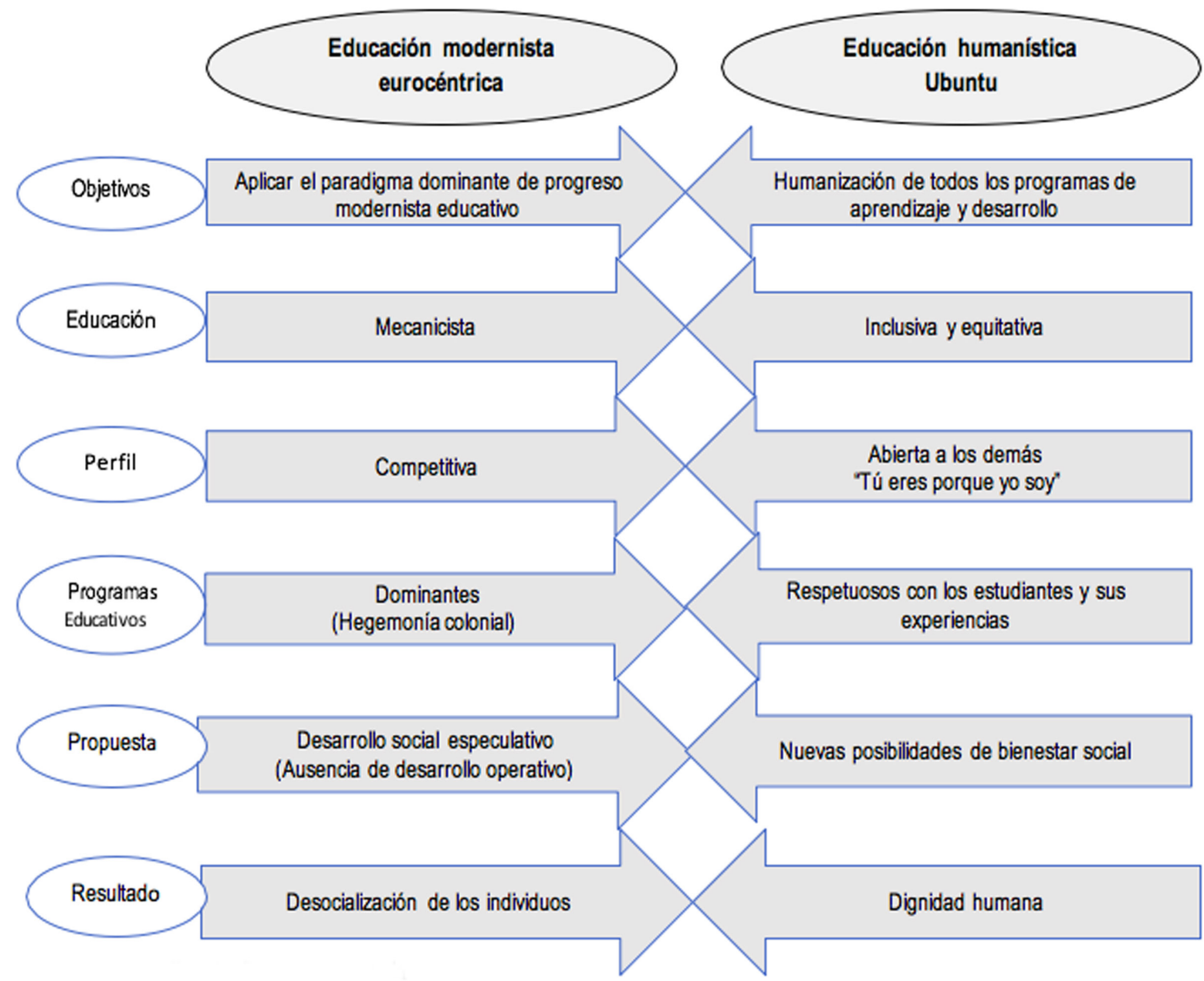

Fuente: Elaboración propia.

En definitiva, la educación humanística de Ubuntu, pretende recuperar historias usurpadas y culturas anuladas por el peso colonial. Intenta mostrar que, la utilización de la educación para crear el «ideal de persona» según las tesis imperialista, ha sido y es, un planteamiento erróneo ávido de injusticia social. Su conocimiento nos va a permitir que los focos investigadores iluminen espacios emergentes, posibilidades de ajustes epistemológicos en la ciencia comparada, que no anulan los preceptos existentes. Ubuntu puede impactar en el campo que nos ocupa, que tiene la capacidad de cambiar el enfoque educativo prevaleciente:

El punto es que existe un marco teórico y cultural que, si se aplica, tanto en África como a nivel mundial, puede considerarse universalmente aceptable y beneficioso. Infundir en la globalización que hasta ahora se alimenta de la dependencia, la marginación y la "codicia» la noción de nutrir el bienestar colectivo o sembrar las semillas de la posibilidad real de colapso colectivo parece crucial (Assié-Lumumba 2016b, p.16).

\section{DISCUSIÓN Y CONCLUSIONES}

A lo largo de este trabajo, hemos pretendido conocer los postulados de un paradigma filosófico que, si bien es originario de África, perfectamente pueden sus principios presidir una educación humanística e incluso, servir de base para el desarrollo epistemológico del campo de la $\mathrm{ECl}$. Recordemos que nos encontramos en un momento en el que, la $\mathrm{ECl}$ sigue siendo objeto de intensos debates, a partir de los cuales se cuestiona su sentido y orientación como ciencia, al mismo tiempo que se destacan los desafíos a los que se enfrenta en una sociedad globalizada.

Es de sobra conocido que el fenómeno de la globalización ha repercutido directamente en los patrones de difusión del conocimiento. El legado epistemológico del colonialismo dibuja el empobrecimien- 
to de las ciencias sociales que se ven evocadas a su revisión a fin de cuenta de poder enfrentarse a los desafíos de carácter social y cultural inducidos por el propi fenómeno.

Los paradigmas dominantes han definido su propio concepto de aprendizaje y de desarrollo a la vez que, han normalizado las teorías y prácticas occidentales como patrón explicativo de las dinámicas mundiales, desnormalizando y ocultando todo aquello que se escapara de ese marco.

En toda esta maraña, se presenta el paradigma Ubuntu, como una perspectiva de conocimiento indígena africano, a partir del cual, se pretende mirar hacia el diálogo entre los pueblos y civilizaciones así como la búsqueda de un consenso en la convergencias de ideas, sumar la sabiduría colectiva que, en ningún caso va a significar jerarquía. Pretende, mostrar a los trabajadores del campo de la $\mathrm{ECl}$, que otras vías de búsqueda de conocimiento son posibles, y no sólo viables, si no necesarias. Que la búsqueda de comparación debe ir más allá de los preceptos establecidos hasta ahora. Que es bueno que los comparativistas se cuestionen ¿qué están comparando y dónde lo están haciendo? Un análisis crítico que insta al cambio de las formas de trabajo y la interpretación de resultados.

\section{REFERENCIAS BIBLIOGRÁFICAS}

Abdi, A. A. (2015). Ubuntu as a platform for humanist education: An introduction. Recuperado de: http://cies2015.org/response-abdi.html.

Abdi A. A. (2018). The Humanist African Philosophy of Ubuntu: Anti-colonial Historical and Educational Analyses. In: Takyi-Amoako E., Assié-Lumumba N. (eds) Re-Visioning Education in Africa: Ubuntu-Inspired Education for Humanity (pp.18-34). Palgrave Macmillan.

Acosta, F y Ruiz, G. (2015). Estudio introductorio. En G.R. Ruiz, y F. Acosta. (eds). Repensando la educación comparada: lecturas desde Iberoamérica. Entre los viajeros del siglo XIX y la globalización (pp.15-26). Barcelona: Octaedro.

Assié-Lumumba, N. T. (2016a). Evolving African attitudes to European education: Resistance, pervert effects of the single system paradox, and the ubuntu framework for renewal. International Review of Education,62 (1), 11-27.

Assié-Lumumba, N. T (2016b). The Ubuntu Paradigm and Comparative and International Education: Epistemological Challenges and Opportunities in Our Field. Comparative Education Review, 61(1), 1-21.

Bray, M. (2010). Actores y propósitos en Educación Comparada. En M. Bray; B. Adamson \& M. Mason. Educación Comparada. Enfoques y Métodos (pp. 39-64). Buenos Aires: Granica.

Botero Gómez, P. (2015). Subjetividades colectivas y prácticas de paz en contextos de guerra. Una perspectiva desde la psicología política decolonial. Prospectiva. Revista de Trabajo Social e Intervención Social, 20, 71-90.

Collado Ruano, J. (2016). Epistemología del Sur: una visión descolonial a los Objetivos de Desarrollo Sostenible. Sankofa. Revista de História da África e de Estudos da Diáspora Africana Ano IX, N. ${ }^{\circ}$ XVII, 137-158.

Cárdenas Oñate, M. (2016). Ubuntu Voz palenquera de múltiples colores Hacia una argumentación metafórica cimarrona. Discurso \& Sociedad, 10 (4), 559-587.

Carnoy, Martin. 2006. «Rethinking the Comparative—and the International.»Comparative Education Review 50 (4): 551-70.

Cillerss, J. (2017). Ubuntu or into? South African perspectives of preaching. In J. Dreyer, Y, Dreyer, E, Foley, \& M. Nel. (eds.). Practicing Ubuntu. Practical theological perspectives on Injustice, Personhood and Human Dignity, pp.67-77. Zürich: LIT VERLANG GmbH\& Co.

Espejo, L. B, Beltrán, JC, Lázaro, L y García, E. (2014). Preface. L. Vega Gil. (Ed.) Empires, Post-Coloniality and Interculturality (pp. 7-8). The Netherlans: Sense Publishers, CESE.

Figueras, A. (2010). UBUNTO. Sudáfrica. El triunfo de la concordia. Barcelona: Plataforma editorial.

Gade, C. B. N. (2011). The historical development of the written discourses on Ubuntu. South African Journal of Philosophy, 30(3), 303-329.

Gade, C. B. N. (2012). What is Ubuntu? Different interpretations among South Africans of African Descent. South African Journal of Philosophy, 31(3), 484-503.

García Ruiz, M. J. (2012a). Impacto de la globalización y el postmodernismo en la epistemología de la Educación Comparada. Revista Española de Educación Comparada, 20,41-80. 
García Ruiz, M. J. (2012b). La educación comparada ¿es una disciplina moderna o postmoderna? En J.L. García Garrido; M. J. García Ruíz y E. Gavari Starkie. La Educación Comparada en tiempos de globalización (pp. 103-147). Madrid: UNED.

González Marrero, S. (2012). La democracia en Sudáfrica. Revista Española de Ciencia Política. (28), 55-84.

Kakozi Kashindi, J. B. (2011). Una comparación entre «ubuntu» como antología relacional en la filosofía africana bantú y el planteamiento «nosótrico». Su relevancia en estudios sobre afrodescendencia. Europeans Rewiews (5), 46-57. Recuperado de: http://www.ieeiweb.eu/wp- content/uploads/2016/01/n5.pdf.

Larsen, M. (2010). New thinking in Comparative Education. Editorial Introduction. In M. Larsen. (Ed.) New thinking in Comparative Education. Honouring Robert Cowen (pp. 1-14). The Netherlans: Sense Publishers.

López, A. (2008). Retos metodológicos de la educación comparada en la sociedad global. Revista de Universidad y Sociedad del Conocimiento (RUSC), 5, (1), 1-9 Recuperado de: http://www.uoc.edu/rusc/5/1/dt/esp/lopez.pdf.

Lyon, P. (1994). Democratización en Sudáfrica. Anuario Internacional CIBOD, 271-282.

Mason, M. (2010). La comparación de culturas. En M. Bray; B. Adamson \& M. Mason. Educación Comparada. Enfoques y Métodos (pp. 207-247). Buenos Aires: Granica.

Metz, T \& Gaie, J. B. R. (2010). The African ethic of Ubuntu/Botho: implications for research on morality, Journal of Moral Education, (39)3, 273-290, DOI: 10.1080/03057240.2010.497609.

Oviawe, JO. (2016). How to rediscover the ubuntu paradigm in education? International Review of Education, 62, (1), 1-10.

Piper, B. (2016). International education is a broken field: Can ubuntu education bring solutions? International Review of Education, 62 (1), 101-111.

Rui, Y. (2010). La comparación de políticas. En M. Bray; B. Adamson \& M. Mason. Educación Comparada. Enfoques y Métodos (pp. 295-320). Buenos Aires: Granica.

Sayers, H. (2012). Ubuntu! El espíritu de la humanidad. Redescubriendo el arte de vivir juntos en armonía. Omán: Oasis Human Resources Department. Recuperado de: http://www.livingvalues.net/resources/Ubuntu/Ubuntu-spanish.pdf.

Takayama, K; Sriprakash, A \& Connell, R. (2016). Toward a Postcolonial Comparative and International Education. Comparative Education Review, 61 (S1), 1-24. 